\title{
Representations of an Algebra of the Gell-Mann-Dashen Type
}

\author{
M. HAVLÍČEK \\ Faculty of Technical and Nuclear Physics, Czech Technical University, Prague
}

Received December 2, 1968

Abstract. Let $G^{\infty}$ denote the infinitedimensional Lie algebra given by commutation relations $\left[a_{m}^{\alpha}, a_{n}^{\beta}\right]=c_{\gamma}^{\alpha \beta} a_{m+n}^{\gamma}(m, n=0,1, \ldots)$, where $c_{\gamma}^{\alpha \beta}$ are structure constants of the arbitrary centerless Lie algebra. The paper is devoted to the construction of a certain class of skewsymmetric irreducible representations (so called F-representations) of the algebra $G^{\infty}$.

\section{Introduction}

Let $G$ be an arbitrary $r$-dimensional real, centerless Lie algebra with commutation relations

$$
\left[a^{\alpha}, a^{\beta}\right]=c_{\gamma}^{\alpha \beta} a^{\gamma}
$$

$(\alpha, \beta, \gamma=1, \ldots, r)$. We now take the vector space $G^{\infty}$ with basis $a_{m}^{\alpha}$ $\left(m=0,1, \ldots, a_{0}^{\alpha} \equiv a^{\alpha}\right)$ formed by all finite linear combinations of $\alpha_{m}^{\alpha}$ and define the following commutation relations among $a_{m}^{\alpha}$

$$
\left[a_{m}^{\alpha}, a_{n}^{\beta}\right]=c_{\gamma}^{\alpha \beta} a_{m+n}^{\gamma} .
$$

These relations respect $\mathrm{J}_{\mathrm{ACOB}}$ identities so that $G^{\infty}$ forms an infinitedimensional Lie algebra-infinitedimensional extension of $G$.

Algebras extended in this way or in a similar way have a physical application $[1,2]$ and the question of their representations arises.

In the paper [3] finitedimensional representations of the more general Gell-Mann-Dashen "current algebra" are constructed. It may be useful to know more general representations of such algebras.

The presented paper is devoted to the study of the class of irreducible representations by skewsymmetric operators of the algebra $G^{\infty}$ which are, in some way, the direct generalization of the finitedimensional skewsymmetric representations of $G^{\infty}$ (these representations are contained in this class, of course).

The paper is part of a larger work in which representations of a certain infinitedimensional Lie algebra $A\left(P, S U_{3}\right)$ having application in elementary particle physics $[4,5]$ are constructed. 


\section{Representations of the Algebra $\mathbf{G}^{\infty}$}

Consider at first any finitedimensional nontrivial representation of $G^{\infty}$ of the dimension $N$. Then $a_{m}^{\alpha}$ are $N \times N$-matrices and it is clear that there exists such $s<\infty, s=0,1, \ldots$ that

$$
\tau^{i} a_{i}^{\alpha}=0^{\star} ; \quad \alpha=1,2, \ldots, r \Leftrightarrow \tau^{i}=0, \quad i=0,1, \ldots, s
$$

and

$\left(\tau^{i}\right.$ and $\varrho_{s+1}^{i}$-real coefficients).

$$
a_{s+1}^{\alpha}=\varrho_{s+1}^{i} a_{i}^{\alpha}
$$

Indeed, in the opposite case the equivalence (3) holds for every $s$. Because equation $\tau^{i} a_{i}^{\alpha}=0$ represents the system at most $2 N^{2} . r$ scalar equations of $s+1$ variables $\tau^{i}$ there exists for $s+1>2 N^{2} r$ the nontrivial solution and it is a contradiction.

Assume that $a_{m}^{\alpha}=\varrho_{m}^{i} a_{i}^{\alpha}$ for some $m$ ( $\varrho_{m}^{i}$-real coefficients). Then from commutation relations (2) we have

$$
c_{\gamma}^{\alpha \beta}\left(\varrho_{m}^{i} a_{i+1}^{\gamma}-a_{m+1}^{\gamma}\right)=0 .
$$

Because algebra $G$ has the trivial center this system of equations has only a trivial solution. It shows, together with Eq. (4) that

$$
a_{m}^{\alpha}=\varrho_{m}^{i} a_{i}^{\alpha}
$$

for every $m$, where $\varrho_{m}^{i}$-real coefficients and $\varrho_{m}^{i}=\delta_{m}^{i}$ for $m \leqq s$.

In this paper we shall study irreducible skewsymmetric representations of $G^{\infty}$ which fulfil conditions (5) without limitation of the dimension ${ }^{1}$. These representations we shall call $F$-representations of $G^{\infty}$.

Substituting (5) into Eq. (2), using triviality of the center of $G$ and Eq. (3) we obtain

$$
\varrho_{m}^{i} \varrho_{n}^{k} \varrho_{i+k}^{l}=\varrho_{m+n}^{l}, \varrho_{k}^{i}=\delta_{k}^{i} .
$$

Eq. (2) shows further that the operators $a_{i}^{\alpha}$ form the representation of the basis of the algebra

$$
\left[a_{i}^{\alpha}, a_{k}^{\beta}\right]=c_{\gamma}^{\alpha \beta} \varrho_{i+k}^{l} a_{l}^{\gamma} .
$$

This algebra we denote $G^{s+1}\left(\varrho_{m}^{i}\right)$ and its dimension equals to $r(s+1)$.

* We use Ernsters's summation convention and in this chapter indices $i, j$, $k, l$, run over 0 to $s$.

1 Note in this connection some fundamental definitions. Following e.g. [6] a representation $l$ of an abstract Lie algebra $L$ by the linear operators in the Hilbert space $H$ is any homomorphism $T$ of $L$ into the set of linear operators in $H L \rightarrow T(L)$ $=l$ having a common dense domain $D_{l}$, invariant with respect to all operators of $l$, on which

$$
T\left(\left[L_{\alpha}, L_{\beta}\right]\right) \cdot \varphi=\left(T\left(L_{\alpha}\right) T\left(L_{\beta}\right)-T\left(L_{\beta}\right) T\left(L_{\alpha}\right)\right) \cdot \varphi
$$

for every $\varphi \in D_{l}$ and $L_{\alpha}, L_{\beta} \in L$. A representation is called skewsymmetric if the homomorphism $T$ maps $L$ into the set of skewsymmetric operators. A representation $l$ on $D_{l}$ is called irreducible if there exists no proper closed subspace $H^{\prime} \subset H$ with a dense set $D^{\prime} \subset H^{\prime}$ such that $l D^{\prime} \subset H^{\prime}$. 
The following theorem summarizes and slightly enlarges the obtained results.

Theorem 1. Every F-representation of the algebra $G^{\infty}$ is determined through Eq. (5) by a certain skewsymmetric irreducible representation of the algebra $G^{s+1}\left(\varrho_{m}^{i}\right)$ where $\varrho_{m}^{i}$ fulfils the condition $(6)$.

Proof of this theorem either follows from the above considerations or is quite clear.

\section{The Structure of the Algebra $G^{s+1}\left(\varrho_{m}^{i}\right)$}

At first we shall consider conditions (6). We introduce $s+1$ dimensional matrices

$$
\Omega_{m}=\left\|\varrho_{m+k}^{i}\right\|, \Omega_{1} \equiv \Gamma, \Omega_{0} \equiv E_{s+1}
$$

$\left(E_{s+1}\right.$ the $(s+1)$-dimensional unit matrix). Using Eq. (6) for $n=1$ $(m \rightarrow m+k)$ we prove

$$
\Omega_{m}=(\Gamma)^{m}
$$

(Here $m$ is exponent. Heretofore $(x)^{m}$ signifies the $m$-th power of $x$ as opposed to $x^{m}$ where $m$ is a superscript.) From this we get the dependence of the constants $\varrho_{m}^{i} m>s+1$ on the constants $\varrho_{s+1}^{i}$ and Eq. (6) is fulfilled identically. In fact from $(8-9)$ we have

$$
\Omega_{a+b}=(\Gamma)^{a}(\Gamma)^{b} \Rightarrow \varrho_{a+b+j}^{l}=\varrho_{b+j}^{k} \varrho_{a+k}^{l}
$$

$(a, b=0,1, \ldots$,$) . If a=i$ and $b+j=n$ in (10) and if we substitute (10) into Eq. (6) we obtain the relation

$$
\varrho_{m}^{i} \varrho_{n+i}^{l}=\varrho_{m+n}^{l} .
$$

This relation is fulfilled because it is (10) for $b+j=m, a=n$ and the discussion of the conditions (6) is finished.

From (10) by substitution $b+j=m, a=i$ we obtain also the relation

$$
(\Gamma)^{m}=\sum_{k=0}^{s} \varrho_{m}^{k}(\Gamma)^{k}
$$

This relation for $m=s+1$ is the well-known Hamilton-Caley relation.

We can easily prove [using Eq. (11)] that $r(s+1)$-dimensional matrices

$$
\operatorname{ad} \alpha_{i}^{\alpha}=C^{\alpha} \times(\Gamma)^{i}
$$

(where $\times$-Kronecker product, $C^{\alpha}$-matrices of the adjoint representation of the algebra $G$ ) form the adjoint representation of the basis of the algebra $G^{s+1}\left(\varrho_{m}^{i}\right)$.

This representation is faithful because the matrices $(\Gamma)^{0} \ldots,(\Gamma)^{s}$ are linearly independent and the adjoint representation of $G$ is faithful because of the triviality of the center of $G$. 
Let $\Gamma$ be any $(s+1) \cdot(s+1)$-matrix, so that the powers $(\Gamma)^{0} \ldots,(\Gamma)^{s}$ are linearly independent ( $T$ is a cyclic matrix). Denote by $G^{s+1}(\Gamma)$ $(s+1) \cdot r$-dimensional Lie algebra with adjoint representation (12), so that $G^{s+1}\left(\varrho_{m}^{i}\right) \equiv G^{s+1}(\Gamma)$. It is clear that, if $\Gamma$ and $\Gamma^{\prime}$ are equivalent matrices, then $G^{s+1}(\Gamma)$ and $G^{s+1}\left(\Gamma^{\prime}\right)$ are isomorphic algebras and we can put $G^{s+1}(\Gamma) \equiv G^{s+1}\left(\Gamma^{\prime}\right)$.

Let $\lambda_{1}, \ldots, \lambda_{p}(p \geqq 0)$ denote all mutually different real roots of characteristic equation of $\Gamma$ with corresponding multiplicities $s_{1}, \ldots, s_{p}$ and let $\lambda_{p+1}, \ldots, \lambda_{p+q}, \bar{\lambda}_{p+1}, \ldots, \bar{\lambda}_{p+q}(q \geqq 0)$ denote all mutually different complex roots of this equation with multiplicities $s_{p+1}, \ldots, s_{p+q}$ so that

Let further

$$
\sum_{a=1}^{p} s_{a}+2 \sum_{a=p+1}^{p+q} s_{a}=s+1
$$

$$
\Gamma^{\prime}=\operatorname{diag}\left\|\Gamma_{1}, \ldots, \Gamma_{p+q}\right\|=\Gamma_{1} \oplus \ldots \oplus \Gamma_{p+q}
$$

denotes the quasidiagonal $(s+1) \cdot(s+1)$-matrix, where for $a=1, \ldots, p$

$$
\Gamma_{a}=\left|\begin{array}{ccccc}
\lambda_{a} & 1 & 0 & \ldots & 0 \\
0 & \lambda_{a} & 1 & \ldots & 0 \\
- & - & - & - & - \\
0 & \ldots & \ldots & \lambda_{a}
\end{array}\right|
$$

and $\Gamma_{a}$ is $s_{a} \cdot s_{a}$-matrix, for $a=p+1, \ldots, p+q$

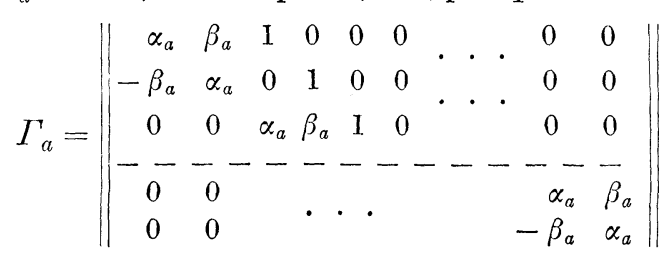

$\left(\alpha_{a}=\operatorname{Re} \lambda_{a}, \beta_{a}=\operatorname{Im} \lambda_{a}\right)$ and $\Gamma_{a}$ is $\left(2 s_{a}\right) \cdot\left(2 s_{a}\right)$-matrix.

It is easy to verify that matrices $\Gamma$ and $\Gamma^{\prime}$ are equivalent (in the real way!) because they have the same elementary divisiors. Eqs. (12) $\left(\Gamma \rightarrow \Gamma^{\prime}\right)$ and (13) give for the basis of $G^{s+1}\left(\Gamma^{\prime}\right) \equiv G^{s+1}(\Gamma)$ the relation

$$
\operatorname{ad} a_{i}^{\alpha}=C^{\alpha} \times\left(\Gamma^{\prime}\right)^{i}=\bigoplus_{a=1}^{p+q} C^{\alpha} \times\left(\Gamma_{a}\right)^{i} .
$$

The powers $\left(\Gamma_{a}\right)^{0}, \ldots\left(\Gamma_{a}\right)^{s_{a}-1}$ for $a=1, \ldots, p$ or $\left(\Gamma_{a}\right)^{0}, \ldots,\left(\Gamma_{a}\right)^{2 s_{a}-1}$ for $a=p+1, \ldots, p+q$ respectively are linearly independent and, due to Hamilton-Caley equation for matrices $\Gamma_{a}$, further powers of $\Gamma_{a}$ are already expressed by means of these initial powers ${ }^{2}$.

$2 \quad$ Assume, on the contrary, that for some $a$ the number of linearly independent powers of the matrix $\Gamma_{a}$ is less than $s_{a}\left(2 s_{a}\right)$. Then the initial $s+1$ linearly independent powers $\left(\Gamma^{\prime}\right)^{0}, \ldots,\left(\Gamma^{\prime}\right)^{s}$ of the matrix $T^{\prime}$ would be expressed as a linear combination of the linearly independent matrices, the number of which is lower than $s+1$. But it is not possible. 
The consequence of this fact and of Eq. (16) is that the algebra $G^{s+1}(T)$ is equal the direct sum of its ideals i.e.

$$
G^{s+1}(\Gamma)=\left(\bigoplus_{a=1}^{p} G^{s_{a}}\left(\Gamma_{a}\right)\right) \oplus\left(\bigoplus_{a=p+1}^{p+q} G^{2 s_{a}}\left(\Gamma_{a}\right)\right) .
$$

We see that in the direct sum we have two fundamental types of ideals according to the two different types of matrices $\Gamma_{a}$. In the following $\Gamma_{a}$ denotes a matrix of the type (14) and $\tilde{\Gamma}_{a}$ denotes a matrix of the type (15).

\section{a) Structure of the Algebra $G^{s_{a}}\left(\Gamma_{a}\right)$}

In this case we can write

where for $s_{a}>1$

$$
\Gamma_{a}=\lambda_{a} E_{s_{a}}+N_{s_{a}}
$$

$$
N_{s_{a}}=\left\|\delta_{i, j-1}\right\|
$$

is $s_{a} \cdot s_{a}$-nilpotent matrix so that $\left(N_{s_{a}}\right)^{s_{a}}=0$ (for $s_{a}=1 ; N_{s_{a}} \equiv 0$ ).

Consider matrices

$$
\operatorname{ad} a_{a, k}^{\alpha^{\prime}}=C^{\alpha} \times\left(N_{s_{a}}\right)^{k}
$$

$\left(k=0, \ldots, s_{a}-1\right)$. These matrices form the basis of the adjoint representation of $G^{s_{a}}\left(\Gamma_{a}\right)$ because of its linear independence and connection with the initial basis in the following way ${ }^{3}$

$$
\operatorname{ad} a_{a, i}^{\alpha}=C^{\alpha} \times\left(\lambda_{a} E_{s_{a}}+N_{s_{a}}\right)^{i}=\sum_{k=0}^{i}\left(\begin{array}{l}
i \\
k
\end{array}\right)\left(\lambda_{a}\right)^{i-k} \cdot C^{\alpha} \times\left(N_{s_{a}}\right)^{k}
$$

from which we have

where

$$
\operatorname{ad} \alpha_{a, i}^{\alpha}=\sum_{k=0}^{i} \xi_{i k}^{a} \operatorname{ad} \alpha_{a k}^{\alpha^{\prime}}
$$

$$
\xi_{i k}^{a}=\left(\begin{array}{l}
i \\
k
\end{array}\right)\left(\lambda_{a}\right)^{i-k}
$$

Eq. (18) gives further the commutation relations among $a_{a, i}^{\alpha^{\prime}}$ :

$$
\left[a_{a, i}^{\alpha^{\prime}}, a_{a, j}^{\beta^{\prime}}\right]=\left\{\begin{array}{lll}
c_{\gamma}^{\alpha \beta} & a_{a, i+j}^{\gamma^{\prime}}, & i+j<s_{a} \\
0 & , & i+j \geqq s_{a} .
\end{array}\right.
$$

We see that $G^{s_{a}}\left(\Gamma_{a}\right)$ equals the semidirect sum of the algebra $G$ and nilpotent subalgebra $G^{N}$ with basis $a_{a, i}^{\alpha^{\prime}} i=1,2, \ldots, s_{a}-1$.

For $s_{a}=1, G^{N}$ is trivial; for $s_{a}=2, G^{N}$ equals commutative $r$-dimensional algebra etc. Because the structure of the algebra $G^{s_{a}}\left(\Gamma_{a}\right)$ depends only on $s_{a}$ and does not depend on $\lambda_{a}$ we write $G^{s_{a}}\left(\Gamma_{a}\right) \equiv G^{s_{a}}$.

${ }^{3}$ We put $\left(\lambda_{a}\right)^{0} \equiv 1$ also for $\lambda_{a}=0$ and $\left(N_{s_{a}}\right)^{0} \equiv E_{s_{a}}$ also for $s_{a}=1$. 
b) Structure of the Algebra $G^{2 s_{a}}\left(\tilde{\Gamma}_{a}\right)$

Now we can write [see Eq. (15)]

where

$$
\tilde{\Gamma}=\alpha_{a} E_{s_{a}} \times E_{2}+\beta_{a} E_{s_{a}} \times F_{2}+N_{s_{a}} \times E_{2}
$$

$$
E_{2}=\left\|\begin{array}{ll}
1 & 0 \\
0 & 1
\end{array}\right\|, \quad F_{2}=\left\|\begin{array}{rr}
0 & 1 \\
-1 & 0
\end{array}\right\|
$$

and $\left(F_{2}\right)^{2}=-E_{2}$. Matrices

$$
\operatorname{ad} a_{a, k}^{\alpha^{\prime}}=C^{\alpha} \times\left(N_{s_{a}}\right)^{k} \times E_{2}, \quad \operatorname{ad} b_{a, k}^{\alpha^{\prime}}=C^{\alpha} \times\left(N_{s_{a}}\right)^{k} \times F_{2}
$$

$\left(k=0,1, \ldots, s_{a}-1\right)$ form the basis of the adjoint representation of the $G^{2 s_{a}}\left(\tilde{\Gamma}_{a}\right)$. The connection with the initial basis is the following [see Eqs. (12), (22-24)]:

$$
\operatorname{ad} a_{a, i}^{\alpha}=\sum_{k=0}^{s_{a}-1}\left(\zeta_{i k}^{a} \operatorname{ad} a_{a, k}^{\alpha^{\prime}}+\eta_{i k}^{a} \operatorname{ad} b_{a, k}^{\alpha^{\prime}}\right), \quad i=0, \ldots, 2 s_{a}-1
$$

where

$$
\begin{aligned}
\zeta_{i k}^{a} & =\left(\begin{array}{l}
i \\
k
\end{array}\right) \operatorname{Re}\left(\lambda_{a}\right)^{i-l_{i}}, \eta_{i k}^{a}=\left(\begin{array}{l}
i \\
k
\end{array}\right) \operatorname{Im}\left(\lambda_{a}\right)^{i-k} \\
\left(\lambda_{a}\right. & \left.=\alpha_{a}+\mathrm{i} \beta_{a}\right) .
\end{aligned}
$$

The commutation relations among $a_{a, k}^{\alpha^{\prime}}$ and $b_{a, k}^{\alpha^{\prime}}$ we obtain from Eq. (24)

$$
\begin{aligned}
& {\left[a_{a, k}^{\alpha^{\prime}}, a_{a, l}^{\beta^{\prime}}\right]=\left\{\begin{array}{cc}
c_{\gamma}^{\alpha \beta} a_{a, k+l}^{\gamma^{\prime}}, & k+l<s_{a} \\
0, & k+l \geqq s_{a}
\end{array} ;\right.} \\
& {\left[a_{a, k}^{\alpha^{\prime}}, b_{a, l}^{\beta^{\prime}}\right]=\left\{\begin{array}{cc}
c_{\gamma}^{\alpha \beta} b_{a, k+l}^{\gamma^{\prime}}, & k+l<s_{a} \\
0, & k+l \geqq s_{a} ;
\end{array}\right.} \\
& {\left[b_{a, k}^{\alpha^{\prime}}, b_{a, l}^{\beta^{\prime}}\right]=\left\{\begin{array}{cc}
-c_{\gamma}^{\alpha \beta} a_{a, k+l}^{\gamma^{\prime}}, & k+l<s_{a} \\
0, & k+l \geqq s_{a} .
\end{array}\right.}
\end{aligned}
$$

These commutation relations describe the real form of the complexification of the algebra $G^{s a}$. For example if $G \equiv S U(2)$ and $s_{a}=1$ then relations (27) are commutative relations of $S L(2, C)$ etc.

As in the preceding case we write $G^{2 s_{a}}\left(\tilde{\Gamma}_{a}\right) \equiv \tilde{G}^{2 s_{a}}$.

\section{The F-Representations of the Algebra $G^{\infty}$-Continuation}

Now we are in a position to formulate the final theorem:

Theorem 2. a) Every irreducible skewsymmetric representation of the algebra

$$
\left(\bigoplus_{a=1}^{p} G^{s_{a}}\right) \oplus\left(\bigoplus_{a=p+1}^{p+q} \tilde{G}^{2 s_{a}}\right)
$$


$\left(s_{a}-1, p, q=0,1, \ldots\right)$ and every set of numbers $\lambda_{1}, \ldots, \lambda_{p+q}\left(\lambda_{a} \neq \lambda_{b}\right.$ for $a \neq b, \lambda_{a}=\bar{\lambda}_{a}$ for $a \leqq p, \lambda_{a} \neq \bar{\lambda}_{a}$ for $a>p$ ) determine through equation

$$
a_{m}^{\alpha}=\sum_{a=1}^{p} \sum_{j=0}^{s_{a}-1} \xi_{m j}^{a} \alpha_{a, j}^{\alpha^{\prime}}+\sum_{a=p+1}^{p+q} \sum_{j=0}^{s_{a}-1}\left(\zeta_{m j}^{a} a_{a, j}^{\alpha^{\prime}}+\eta_{m j}^{a} b_{a, j}^{\alpha^{\prime}}\right)
$$

( $a_{a, j}^{\alpha^{\prime}}, a \leqq p$ resp. $a_{a, j}^{\alpha^{\prime}}, b_{a, j}^{\alpha^{\prime}} a>p$-generators of $G^{s_{a}}$ or $G^{2 s_{a}}$ respectively; coefficients are defined by Eqs. (20) and (26)) a F-representation of $G^{\infty}$.

b) In this way we obtain the set of all F-representations of $G^{\infty}$.

Proof. a) This part of the theorem, except for the irreducibility of the representation of $G^{\infty}$, we easily prove by direct calculation, using Eqs. $(20-1),(26-8)$ and the general formula

$$
\left(\begin{array}{c}
m+n \\
k
\end{array}\right)=\sum_{j=0}^{k}\left(\begin{array}{c}
m \\
j
\end{array}\right)\left(\begin{array}{c}
n \\
k-j
\end{array}\right) .
$$

We prove irreducibility of the representation (29) of the algebra $G^{\infty}$. The transformation (29) connecting the first $r(s+1)$ operators of the algebra $G^{\infty}$ with operators of the algebra (28) is regular. To prove it, consider the adjoint representation of $G^{s+1}\left(\Gamma^{\prime}\right)$ with $\Gamma^{\prime}$ given by Eqs. (13-5). Substituting from Eqs. (17) and (22) into Eq. (16), using Eqs. (18), (24) and further Eqs. (20), (26) we obtain

$$
\operatorname{ad} a_{i}^{\alpha}=\bigoplus_{a=1}^{p} \sum_{j=0}^{s_{a}-1} \xi_{i, j}^{a} \operatorname{add} \alpha_{a, j}^{\alpha^{\prime}} \oplus\left(\bigoplus_{a=p+1}^{p+q} \sum_{j=0}^{s_{a}-1}\left(\zeta_{i j}^{a} \operatorname{ad} a_{a, j}^{\alpha^{\prime}}+\eta_{i j}^{a} \operatorname{ad} b_{a, j}^{\alpha^{\prime}}\right)\right) .
$$

Because the matrices of the right side of Eq. (30) form a linearly independent set with the $r(s+1)$ elements and the same also holds for the matrices ad $\alpha_{i}^{\alpha}$, the transformation connecting them is regular. It is exactly the transformation (29) for $m \leqq s$. Therefore we can calculate $a_{a, j}^{\alpha^{\prime}} \in G^{s_{a}}$ and $\alpha_{a, j}^{\alpha^{\prime}}, b_{a, j}^{\alpha^{\prime}} \in \widetilde{G}^{2 s_{a}}$ by means of $\alpha_{i}^{\alpha} i \leqq s$ and reducibility with respect to these operators $a_{i}^{\alpha}$ implies reducibility with respect to algebra (28) which contradicts our assumption.

b) For every $F$-representation of $G^{\infty}$, following Theorem 1, there exists a skewsymmetric irreducible representation of $G^{s+1}(I)$ with certain $s$ and $\Gamma$, such that the operators $a_{m}^{\alpha} \in G^{\infty}$ are expressed with help of $a_{i}^{\alpha} \in G^{s+1}(\Gamma)$ through Eq. (5). The connection of $a_{i}^{\alpha} \in G^{s+1}(\Gamma)$ with generators $a_{a, j}^{\alpha^{\prime}} \in G^{s_{a}}$ and $a_{a, i}^{\alpha^{\prime}}, b_{a, j}^{\alpha^{\prime}} \in G^{2 s_{a}}$ is given by Eq. (30) (ad $a_{a, i}^{\alpha^{\prime}} \rightarrow a_{a, i}^{\alpha^{\prime}}$ etc). Further, for every eigenvalue $\lambda_{a}$ of the matrix $\Gamma$, the relation holds

$$
\left(\begin{array}{c}
m \\
j
\end{array}\right)\left(\lambda_{a}\right)^{m}=\sum_{i=0}^{s} \varrho_{m}^{i}\left(\begin{array}{l}
i \\
j
\end{array}\right)\left(\lambda_{a}\right)^{i}
$$

$\left(j=0, \ldots, s_{a}-1\right)$. Actually, the matrix $\Gamma$ is equivalent (in the complex way) to the canonical Jordan form i.e. equals the direct sum of the matrices of the type (14) with possible complex $\lambda_{a}$. Substituting this 
form of $\Gamma$ in Eq. (11) we easily obtain the desired result. The direct consequence of the relation (31) are the relations

$$
\xi_{m j}^{a}=\sum_{i=0}^{s_{a}} \varrho_{m}^{i} \xi_{i j}^{a}, \zeta_{m j}^{a}=\sum_{i=0}^{s_{a}} \varrho_{m}^{i} \zeta_{i j}^{a}, \eta_{m j}^{a}=\sum_{i=0}^{s_{a}} \varrho_{m}^{i} \eta_{i j}^{a}, \quad j=0, \ldots, s_{a}-1 .
$$

Substituting (30) in Eq. (5) and using these relations, we immediately obtain Eq. (29) and the proof of the theorem is finished.

\title{
V. Concluding Remarks
}

a) Let $G$ be a compact algebra and $s_{1}=\ldots=s_{p}=1, q=0$ in the relation (28). Then the algebra (28) equals

$$
\underbrace{G \oplus \ldots \oplus G}_{p \text {-times }}
$$

because $G^{1} \equiv G$ [see Eq. (21)] and relation (29) leads to

$$
a_{m}^{\alpha}=\sum_{a=1}^{p}\left(\lambda_{a}\right)^{m} a_{a}^{\alpha} \quad\left(a_{a, 0}^{\alpha} \equiv a_{a}^{\alpha}\right)
$$

In this case the algebra (28) is compact and has nontrivial finitedimensional skewsymmetric representations, so that the $F$-representation of $G^{\infty}$ is also finitedimensional.

b) Beyond these finitedimensional $F$-representations of $G^{\infty}$, there exists for compact $G$ the set of $F$-representations of infinite dimension. For example, if $G \equiv S U_{2}$ algebra (28) can be the algebra of the Euclidian group of motions in three dimensions $\left(p=1, s_{1}=2, q=0\right)$ or algebra $S L(2, C)\left(p=0, q=1, s_{1}=1\right)$ etc.

Acknowledgements: The author would like to thank J. VotruBA for the valuable comments and discussions and to J. ForMANEK for critically reading an earlier draft of the present paper.

\section{References}

1. JERsák, J., and J. Štern: Relativistic non-invariance symmetries generated by local currents. Preprint of the Charles University, Prague, March 1968. To be published in Nucl. Phys.

2. Dashen, R. F., and M. Gell-mand: Phys. Rev. Letters 17, 340 (1966).

3. Roffman, E. H.: J. Math. Phys. 8, 1954 (1967).

4. Formánek, J.: Nucl. Phys. 87, 376 (1966).

5. Votruba, J., and M. HAVLíčEK: Fizika vysokich energij i teorija elementarnych častic p. 330. Kiev: Naukova dumka 1967.

6. Doebner, H. D., and O. Melshemere: Nuovo Cimento 10, 73 (1967).

\author{
M. HAVLÍČEK \\ Faculty of Technical \\ and Nuclear Physics \\ Brehova 7 \\ Prague 1, CSSR
}

